\title{
Recent Trends in Creatinine Assays in Korea: Long- Term Accuracy-Based Proficiency Testing Survey Data by the Korean Association of External Quality Assessment Service (2011-2019)
}

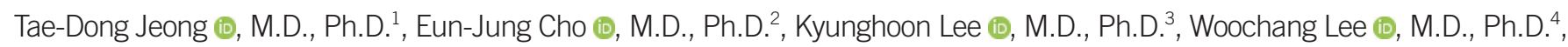

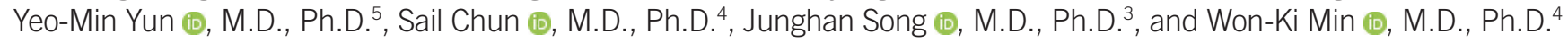

${ }^{1}$ Department of Laboratory Medicine, Ewha Womans University College of Medicine, Seoul, Korea; ${ }^{2}$ Department of Laboratory Medicine, Hallym University Dongtan Sacred Heart Hospital, Hallym University College of Medicine, Hwaseong, Korea; ${ }^{3}$ Department of Laboratory Medicine, Seoul National University Bundang Hospital and College of Medicine, Seongnam, Korea; ${ }^{4}$ Department of Laboratory Medicine, University of Ulsan College of Medicine and Asan Medical Center, Seoul, Korea; ${ }^{5}$ Department of Laboratory Medicine, Konkuk University School of Medicine, Konkuk University Medical Center, Seoul, Korea

Background: Accurate serum creatinine (Cr) concentration measurement is essential for evaluating kidney function. In 2011, the Korean Association of External Quality Assessment Service (KEQAS) launched an accuracy-based Cr proficiency testing (ABCr PT) survey. We analyzed long-term data of the KEQAS ABCr PT survey collected between 2011 and 2019 to assess recent trends in Cr assays in Korea.

Methods: The ABCr PT survey including three commutable fresh-frozen serum samples was performed twice a year. The target $\mathrm{Cr}$ concentration was assigned using isotope-dilution mass spectrometry. We analyzed data obtained from the participating laboratories, calculated the yearly bias, and evaluated bias trends for the major reagents and instruments. Outliers were excluded from all analysis.

Results: The mean percentage bias based on the total data of all participating laboratories was $10.8 \%$ in the 2011 -A survey and $0.2 \%$ in 2019-B survey. Bias for the major reagents and instruments differed depending on the manufacturer. Enzymatic assays generally showed desirable bias ranging from $-3.9 \%$ to $3.2 \%$ at all $\mathrm{Cr}$ concentrations and lower interlaboratory variability than non-enzymatic assays (enzymatic vs. non-enzymatic, 3.3\%$7.2 \%$ vs. $6.3 \%-9.1 \%)$.

Conclusions: Although the mean percentage bias of $\mathrm{Cr}$ assays tends to decrease over time, it is necessary to continuously strive to improve $\mathrm{Cr}$ assay accuracy, especially at low concentrations.

Key Words: Accuracy, Bias, Creatinine, Proficiency testing, Survey
Received: July 14, 2020

Revision received: August 17, 2020

Accepted: January 11, 2021

\section{Corresponding author:}

Won-Ki Min, M.D., Ph.D.

Department of Laboratory Medicine, University of Ulsan College of Medicine and Asan Medical Center, 88 Olympic-ro 43-gil, Songpa-gu, Seoul 05505, Korea

Tel: $+82-2-3010-4503$

Fax: +82-2-478-0884

E-mail:wkmin@amc.seoul.kr

\section{INTRODUCTION}

The estimated glomerular filtration rate (eGFR) is the single best indicator for evaluating kidney function [1]. eGFR calculations recommended by organizations such as the Kidney Disease Improving Global Outcomes (KDIGO) and National Kidney Disease
Education Program (NKDEP) are mainly based on serum creatinine $(\mathrm{Cr})$ and cystatin $\mathrm{C}$, and $\mathrm{Cr}$ is widely used in current clinical practice [2]. Cr-based eGFR values form the basis for medical decision making, including diagnosis, classification, prognosis, and determination of treatment policies, for chronic kidney disease and/or acute kidney injury [2-5]. Accurate $\mathrm{Cr}$ concentra- 
tion measurement is essential to maximize the value of the laboratory test data. Clinical laboratories should use a calibrator that is traceable by an isotope dilution mass spectrometry (IDMS) measurement procedure, and the precision and accuracy of $\mathrm{Cr}$ concentration measurement should be continuously monitored through internal quality control and proficiency testing (PT) [6-8].

The Korean Association of External Quality Assessment Service (KEQAS) has been conducting the accuracy-based $\mathrm{Cr}$ PT (ABCr PT) survey since 2011; we previously reported the results of this survey conducted between 2011 and 2017 [9]. The mean percentage bias for all participating laboratories significantly decreased from 11.1\% in 2011 to $2.4 \%$ in 2017 [9]. However, the previous study included outliers, and detailed analyses according to the assay principle (i.e., enzymatic vs. Jaffe method) or target $\mathrm{Cr}$ concentration (i.e., low vs. high) were not performed and their effects on bias were not evaluated. Until 2019, participation in the KEQAS ABCr PT survey was voluntary; however, 2020 onward, all laboratories participating in KEQAS PT have to participate in the ABCr PT survey. More small- to medium-sized laboratories may participate from 2020; however, to date, mainly medium- to large-sized laboratories were included. As laboratory size can affect analytical performance [10], we thought it would be meaningful to analyze bias trends based on KEQAS ABCr PT survey data collected until 2019. We analyzed KEQAS ABCr PT survey data from 2011 to 2019 to identify trends in $\mathrm{Cr}$ assay standardization across participating laboratories and to explore ways to improve the quality of $\mathrm{Cr}$ assays based on longterm ABCr PT survey data.

\section{MATERIALS AND METHODS}

\section{KEQAS ABCr PT survey}

We retrospectively reviewed the KEQAS ABCr PT survey data. This study did not involve human subjects and was exempted from the approval of Institutional Review Board of Ewha Womans University Seoul Hospital. The KEQAS ABCr PT survey was performed twice a year ( $A$ and $B$ trials in sequential order) from 2011 to 2019. Three samples of fresh frozen serum were used in each survey and were processed according to the Clinical \& Laboratory Standards Institute document C37-A, with modification [11]. Venous blood was drawn from donors into plastic blood bags without anticoagulant. Within 5 minutes of blood collection, plasma was separated by centrifugation at $1,500 \times g, 4^{\circ} \mathrm{C}$ for 8 minutes. The plasma was transferred to a clean and sterile borosilicate glass bottle and allowed to clot at room temperature for 4 hours. The serum was separated by centrifugation at 2,700 $\times \mathrm{g}$ for 20 minutes. Cr was spiked to prepare high $\mathrm{Cr}$ concentration serum pools. Serum pools were incubated at $4^{\circ} \mathrm{C}$ for 14 hours under constant, low-speed, magnetic stirrer mixing to ensure homogeneity. The serum pools were then filtered through a $0.22-\mu \mathrm{m}$ hydrophilic membrane and aliquoted into glass vials at $4^{\circ} \mathrm{C}$. The samples were stored at $-70^{\circ} \mathrm{C}$ until shipment to $\mathrm{ABCr}$ PT survey participants. The target $\mathrm{Cr}$ concentration was measured by IDMS in the Reference Material Institute for Clinical Chemistry Standards (Kawasaki, Japan).

\section{Outlier elimination}

The distribution of $\mathrm{Cr}$ data from each participating laboratory was visually observed, and any difference greater than $\pm 50 \%$ from the target $\mathrm{Cr}$ concentration was considered an outlier and excluded from further analyses. Detailed participant responses and the number of outliers are described in Table 1.

\section{Statistical analysis}

To investigate bias (measured $\mathrm{Cr}$ concentration-target $\mathrm{Cr}$ concentration) trends in $\mathrm{Cr}$ assays for all participants from 2011 to 2019, the mean percentage bias and 95\% confidence interval $(\mathrm{Cl})$ for the mean were calculated after outlier elimination. For the bias analysis, samples were classified into three groups based on the target $\mathrm{Cr}$ concentrations $(<88.40 \mu \mathrm{mol} / \mathrm{L}[1.00 \mathrm{mg} / \mathrm{dL}]$, 88.40-175.92 $\mu \mathrm{mol} / \mathrm{L}$ [1.00-1.99 mg/dL], and 176.80-264.32 $\mu \mathrm{mol} / \mathrm{L}[2.00-2.99 \mathrm{mg} / \mathrm{dL}]$ ), and into two groups based on the assay principle (enzymatic vs. non-enzymatic methods) of $\mathrm{Cr}$ concentration measurement. The non-enzymatic assays were subdivided into kinetic Jaffe with compensation, kinetic Jaffe without compensation, and rate-blanked and compensated kinetic Jaffe. The assay principles reported may differ from those used in actual laboratory practice, as they were simply divided into enzymatic and non-enzymatic methods. The interlaboratory coefficient of variation (CV) depending on the $\mathrm{Cr}$ assay principle was calculated. To evaluate the cumulative ABCr PT survey participation effect, bias was analyzed by grouping the responses in order of survey participation. In total, 328 laboratories participated in the ABCr PT survey over nine years. Nine of these laboratories participated only once, and 32 laboratories participated in all 18 surveys. Based on the first bias results, post-hoc analysis was conducted using Dunnett's test. Forest plots were used to identify bias trends for major reagents and instruments that were used by more than 10 participants per trial of the ABCr PT survey.

For statistical analysis, MedCalc Statistical Software version 18.10.2 (MedCalc Software Bvba, Ostend, Belgium) and Anal- 
Table 1. Bias based on KEQAS ABCr PT survey data from 2011 to 2019

\begin{tabular}{|c|c|c|c|c|c|c|c|}
\hline \multirow{2}{*}{ Trial } & \multirow{2}{*}{$\begin{array}{c}\text { Total } \mathrm{N} \text { of } \\
\text { participating labs }\end{array}$} & \multirow{2}{*}{$\begin{array}{l}N \text { of response } \\
\text { results* }\end{array}$} & \multirow{2}{*}{$\begin{array}{c}\mathrm{N} \text { of } \\
\text { outliers }^{\dagger}\end{array}$} & \multicolumn{2}{|c|}{ Bias (\%) } & \multicolumn{2}{|l|}{ Bias (mg/dL) } \\
\hline & & & & Mean $(95 \% \mathrm{Cl})$ & SD & Mean $(95 \% \mathrm{Cl})$ & SD \\
\hline 2011-A & 54 & 161 & 1 & $10.82(9.45-12.19)$ & 12.15 & $0.096(0.080-0.112)$ & 0.102 \\
\hline 2011-B & 56 & 166 & 0 & $7.12(5.77-8.46)$ & 11.02 & $0.075(0.058-0.091)$ & 0.108 \\
\hline $2012-A$ & 103 & 303 & 2 & $9.33(8.33-10.33)$ & 10.79 & $0.082(0.072-0.092)$ & 0.090 \\
\hline 2012-B & 108 & 323 & 0 & $6.18(5.22-7.15)$ & 9.86 & $0.062(0.050-0.073)$ & 0.105 \\
\hline $2013-A$ & 140 & 420 & 1 & $5.78(4.93-6.62)$ & 10.10 & $0.049(0.036-0.061)$ & 0.133 \\
\hline 2013-B & 139 & 416 & 0 & $7.63(6.78-8.48)$ & 10.81 & $0.066(0.057-0.074)$ & 0.091 \\
\hline $2014-A$ & 176 & 527 & 4 & $4.04(3.29-4.80)$ & 11.29 & $0.024(0.013-0.036)$ & 0.132 \\
\hline 2014-B & 167 & 501 & 2 & $-0.01(-0.79-0.76)$ & 7.07 & $-0.004(-0.012-0.004)$ & 0.091 \\
\hline $2015-A$ & 175 & 525 & 1 & $1.36(0.60-2.11)$ & 6.67 & $0.018(0.011-0.026)$ & 0.090 \\
\hline $2015-B$ & 178 & 534 & 1 & $3.64(2.89-4.39)$ & 7.97 & $0.036(0.028-0.045)$ & 0.100 \\
\hline $2016-A$ & 146 & 437 & 3 & $2.37(1.54-3.20)$ & 7.69 & $0.02(0.011-0.029)$ & 0.098 \\
\hline $2016-B$ & 150 & 450 & 2 & $4.01(3.20-4.83)$ & 10.04 & $0.027(0.019-0.036)$ & 0.091 \\
\hline $2017-A$ & 153 & 459 & 3 & $1.77(0.96-2.58)$ & 8.82 & $0.008(0.000-0.015)$ & 0.080 \\
\hline 2017-B & 167 & 501 & 3 & $2.05(1.27-2.82)$ & 8.95 & $0.014(0.008-0.02)$ & 0.068 \\
\hline $2018-A$ & 197 & 591 & 1 & $2.36(1.65-3.07)$ & 8.57 & $0.008(0.000-0.015)$ & 0.093 \\
\hline $2018-B$ & 200 & 600 & 0 & $3.87(3.17-4.58)$ & 8.11 & $0.033(0.027-0.039)$ & 0.074 \\
\hline 2019-A & 227 & 681 & 2 & $0.45(-0.21-1.11)$ & 6.40 & $-0.003(-0.009-0.003)$ & 0.077 \\
\hline 2019-B & 227 & 681 & 2 & $0.20(-0.47-0.86)$ & 7.36 & $-0.018(-0.026-0.009)$ & 0.111 \\
\hline
\end{tabular}

${ }^{*} \mathrm{~N}=3$ samples per trial; ${ }^{\dagger} \mathrm{An}$ outlier was defined as a result with an absolute percentage bias $>50 \%$ of the target $\mathrm{Cr}$ concentration.

Abbreviations: ABCr PT, accuracy-based creatinine proficiency testing; Cr, creatinine; KEQAS, Korean Association of External Quality Assessment Service.

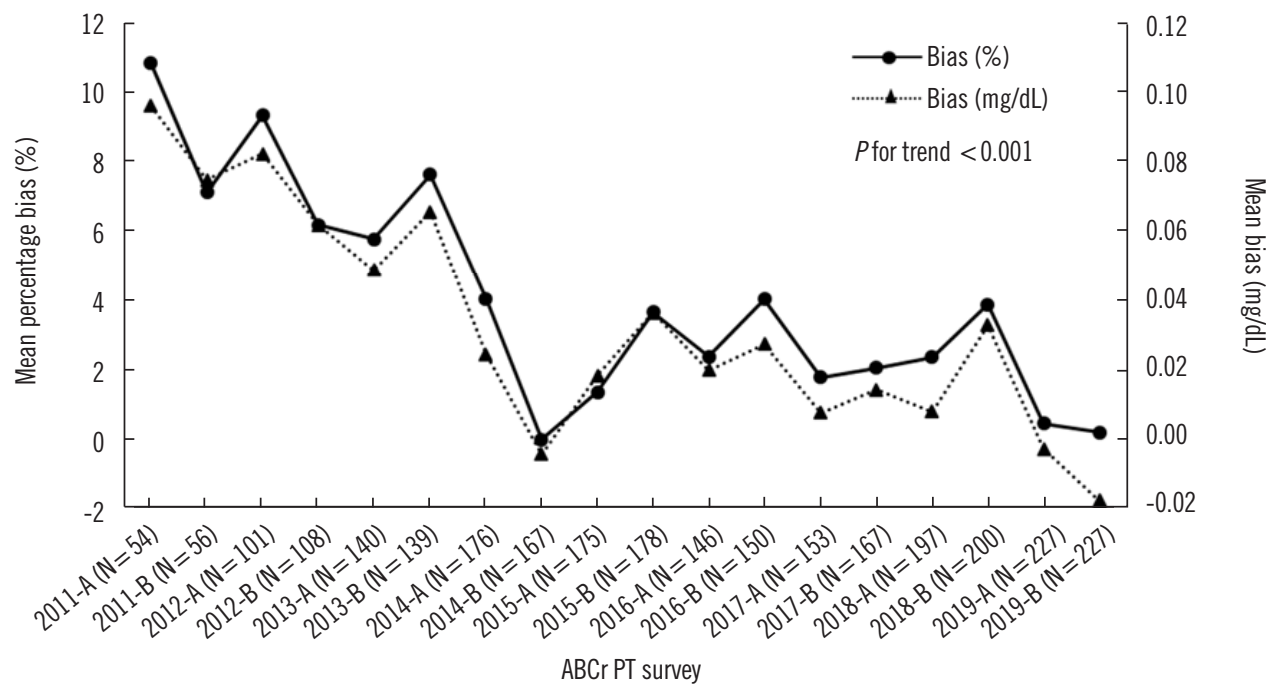

Fig. 1. Trend in mean percentage bias in the ABCr PT survey by the KEQAS (2011-2019). N represents the number of participating laboratories in the first $(A)$ and second $(B) A B C r$ PT survey of the year.

Abbreviations: ABCr PT, accuracy-based creatinine proficiency testing; KEQAS, Korean Association of External Quality Assessment Service.

yse-it for Microsoft Excel 5.51.1 (Analyse-it Software, Leeds, UK) were used. $P<0.05$ was considered statistically significant.

\section{RESULTS}

The mean percentage bias $(95 \% \mathrm{Cls})$ for all participants was 
$10.8 \%(9.5 \%-12.2 \%)$ for $2011-A$, and $0.2 \%(-0.5 \%-0.9 \%)$ for 2019-B. Between 2011 and 2019, the mean percentage bias significantly decreased ( $P$ for trend $<0.001$ ). Detailed mean percentage bias and $95 \% \mathrm{Cls}$ for all $\mathrm{ABCr}$ PT survey data are presented in Fig. 1 and Table 1.

The bias differed depending on the $\mathrm{Cr}$ concentration. For the group with $\mathrm{Cr}$ concentrations $<1.00 \mathrm{mg} / \mathrm{dL}$, the mean percentage bias was $12.8 \%$ in 2011 and 3.5\% in 2019. The group with Cr concentrations of $1.00-1.99 \mathrm{mg} / \mathrm{dL}$ showed $2.5 \%-6.3 \%$ positive bias between 2011 and 2016, and a $\pm 1.5 \%$ decrease after 2017. The group with $\mathrm{Cr}$ concentrations of $2.00-2.99 \mathrm{mg} / \mathrm{dL}$ showed a bias of $\pm 3 \%$ regardless of the year (Fig. $2 A$ ).

The mean percentage bias for enzymatic assays was within $\pm 4 \%$ from 2011 to 2019, and the interlaboratory \%CV was 3.3\%-
Table 2. Interlaboratory percentage coefficient of variations

\begin{tabular}{llcc}
\hline & \multicolumn{3}{c}{ Interlaboratory coefficient of variation (\%) } \\
\cline { 2 - 4 } Year & All & Enzymatic methods & Non-enzymatic methods \\
\hline 2011 & 9.5 & 7.2 & 9.1 \\
2012 & 8.3 & 4.1 & 8.3 \\
2013 & 8.6 & 4.6 & 8.4 \\
2014 & 8.0 & 5.2 & 8.1 \\
2015 & 6.7 & 6.1 & 6.5 \\
2016 & 7.7 & 7.2 & 7.7 \\
2017 & 8.2 & 4.8 & 8.3 \\
2018 & 6.9 & 4.7 & 6.9 \\
2019 & 6.1 & 3.3 & 6.3 \\
\hline
\end{tabular}

A

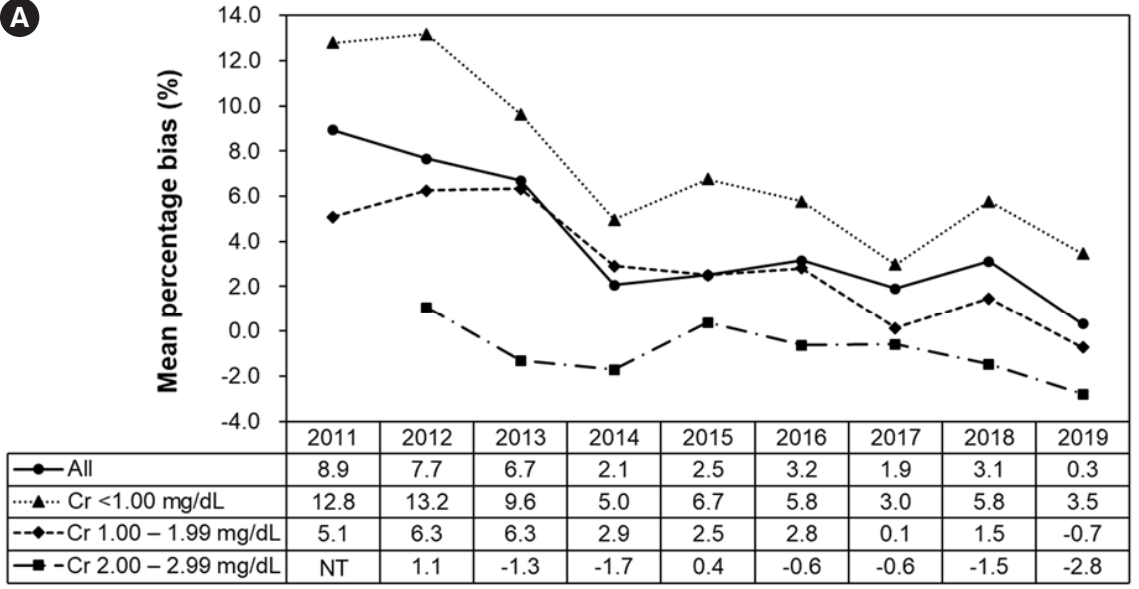

ABCr PT survey

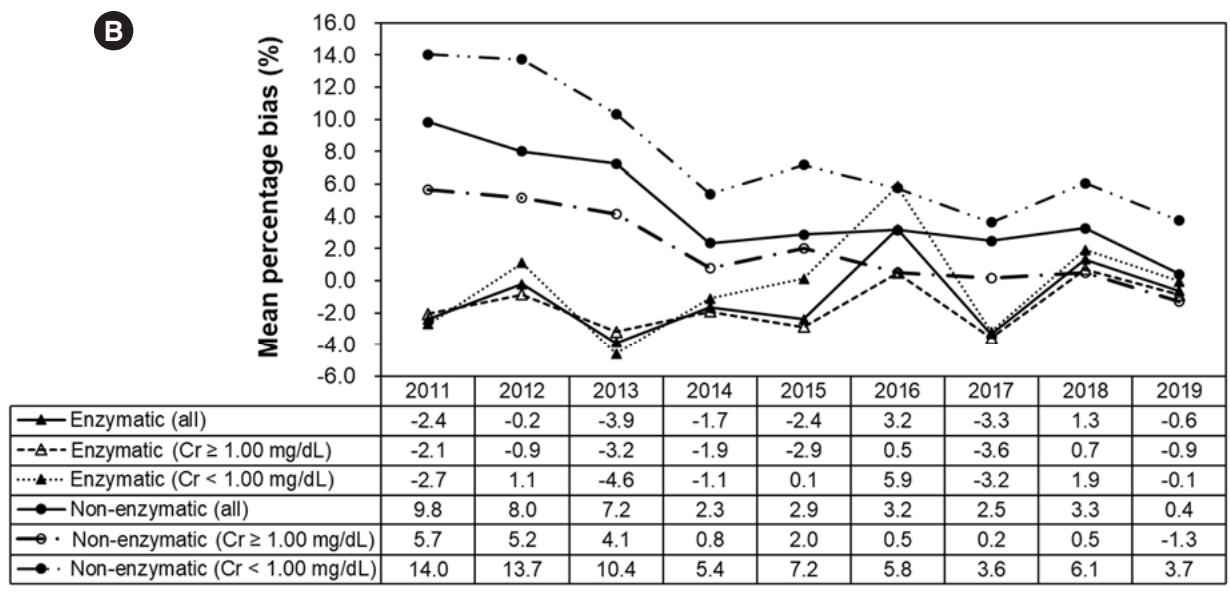

ABCr PT survey

Fig. 2. Mean percentage bias in the ABCr PT survey stratified by (A) Cr concentrations and (B) assay methods. Abbreviations: ABCr PT, accuracy-based creatinine proficiency testing; $\mathrm{Cr}$, creatinine; NT, not tested. 
Table 3. Percentage bias of ABCr PT survey by cumulative trial

\begin{tabular}{|c|c|c|c|c|c|}
\hline \multirow[t]{2}{*}{ Cumulative trial } & \multirow{2}{*}{$\begin{array}{l}\text { Nof participating } \\
\text { laboratories }\end{array}$} & \multicolumn{2}{|c|}{ Bias (\%) } & \multicolumn{2}{|c|}{$\begin{array}{l}\text { Difference in mean percentage bias compared with } \\
\text { the 1st trial* }\end{array}$} \\
\hline & & Mean (95\% Cl) & SD & Mean $(95 \% \mathrm{Cl})$ & $P$ \\
\hline 1 st & 328 & $5.51(4.94-6.08)$ & 10.87 & Reference & \\
\hline 2nd & 319 & $4.74(4.16-5.31)$ & 9.95 & $-0.77(-1.98-0.44)$ & 0.584 \\
\hline 3rd & 268 & $4.31(3.68-4.93)$ & 10.61 & $-1.20(-2.47-0.07)$ & 0.081 \\
\hline 4th & 258 & $3.45(2.80-4.09)$ & 10.24 & $-2.06(-3.35--0.77)$ & $<0.001$ \\
\hline 5th & 206 & $3.29(2.56-4.01)$ & 9.44 & $-2.22(-3.60--0.84)$ & $<0.001$ \\
\hline 6th & 196 & $2.66(1.91-3.40)$ & 9.20 & $-2.85(-4.25--1.45)$ & $<0.001$ \\
\hline 7 th & 172 & $2.60(1.80-3.39)$ & 8.68 & $-2.91(-4.37--1.45)$ & $<0.001$ \\
\hline 8th & 163 & $1.51(0.70-2.33)$ & 7.85 & $-3.99(-5.48--2.50)$ & $<0.001$ \\
\hline 9th & 133 & $2.32(1.42-3.22)$ & 7.14 & $-3.18(-4.78--1.59)$ & $<0.001$ \\
\hline 10th & 128 & $3.08(2.17-4.00)$ & 7.78 & $-2.42(-4.04--0.81)$ & $<0.001$ \\
\hline 11th & 111 & $1.82(0.84-2.79)$ & 6.72 & $-3.69(-5.38--1.99)$ & $<0.001$ \\
\hline 12th & 107 & $2.40(1.40-3.41)$ & 8.03 & $-3.10(-4.83--1.37)$ & $<0.001$ \\
\hline 13th & 88 & $1.86(0.75-2.96)$ & 7.42 & $-3.65(-5.51--1.78)$ & $<0.001$ \\
\hline 14th & 85 & $1.68(0.55-2.81)$ & 7.33 & $-3.83(-5.72--1.93)$ & $<0.001$ \\
\hline 15th & 67 & $1.65(0.37-2.92)$ & 6.27 & $-3.86(-5.95--1.77)$ & $<0.001$ \\
\hline 16th & 60 & $2.16(0.81-3.50)$ & 6.61 & $-3.35(-5.53--1.16)$ & $<0.001$ \\
\hline 17th & 38 & $0.02(-1.67-1.72)$ & 5.36 & $-5.48(-8.15--2.81)$ & $<0.001$ \\
\hline 18th & 32 & $0.24(-1.58-2.07)$ & 7.18 & $-5.26(-8.13--2.40)$ & $<0.001$ \\
\hline
\end{tabular}

*Dunnett's test.

Abbreviations: $\mathrm{ABCr} P \mathrm{PT}$, accuracy-based creatinine proficiency testing; $\mathrm{Cl}$, confidence interval.

$7.2 \%$. The mean percentage bias for non-enzymatic methods was $9.8 \%$ in 2011 and within $\pm 3.5 \%$ since 2014 . The interlaboratory \%CV of non-enzymatic assays was 6.3\%-9.1\% (Fig. 2B and Table 2).

Compared with that in the first participation, the bias significantly decreased after more than four participations (1st vs. 4th, difference in mean percentage bias $-2.06 \%, 95 \% \mathrm{Cl}-3.35 \%-$ $-0.77 \%, P<0.001$ ) (Table 3).

Beckman Coulter reagents showed 6.8\%-7.2\% positive bias in $2011-2013$, <1\% positive bias in 2014-2018, and $-2.5 \%$ negative bias in 2019. Roche reagents had an overall $-0.1 \%-4.1 \%$ positive bias. Sekisui reagents showed $6.0 \%-12.7 \%$ positive bias between 2011 and 2017, which decreased to 2.7\% in 2019. Siemens reagents had $2.1 \%-6.1 \%$ positive bias between 2011 and 2013, and -5.5\% negative bias as of 2014 (Fig. 3A). The bias for Beckman Coulter, Roche, and Siemens instruments was similar to that for the assay reagents. The Hitachi instrument had $4.1 \%-$ 9.7\% positive bias between 2011 and 2017 and 2.6\%-5.1\% in 2018-2019. The Toshiba instrument had a 13.9\% positive bias in 2011, which gradually decreased to $-1.2 \%$ in 2019 (Fig. 3B).

\section{DISCUSSION}

The strength of this study was that the bias trend analysis for $\mathrm{Cr}$ assays was based on KEQAS ABCr PT survey data collected over nine years. The mean percentage bias for all participants, excluding outliers, significantly decreased from $10.8 \%$ for $2011-A$ to $0.2 \%$ for $2019-B$. The decrease in bias was the most prominent for non-enzymatic assays, with $\mathrm{Cr}$ concentrations $<1.0$ $\mathrm{mg} / \mathrm{dL}$. This trend was similar to that in previous studies [10, 12, 13].

For the Beckman Coulter and Siemens platforms, the annual bias had decreased significantly since 2014. This is mainly because an IDMS-traceable calibrator was used in the participating laboratories. Standardized $\mathrm{Cr}$ assays with an IDMS-traceable calibrator yielded $\mathrm{Cr}$ concentrations $0.1-0.2 \mathrm{mg} / \mathrm{dL}$ lower than non-IDMS-traceable assays, indicating that only changes in the calibrator can be expected to improve positive bias in $\mathrm{Cr}$ assays $[14,15]$.

Constant positive bias, probably due to a systematic error, was observed for the Sekisui reagents. The bias especially increased 

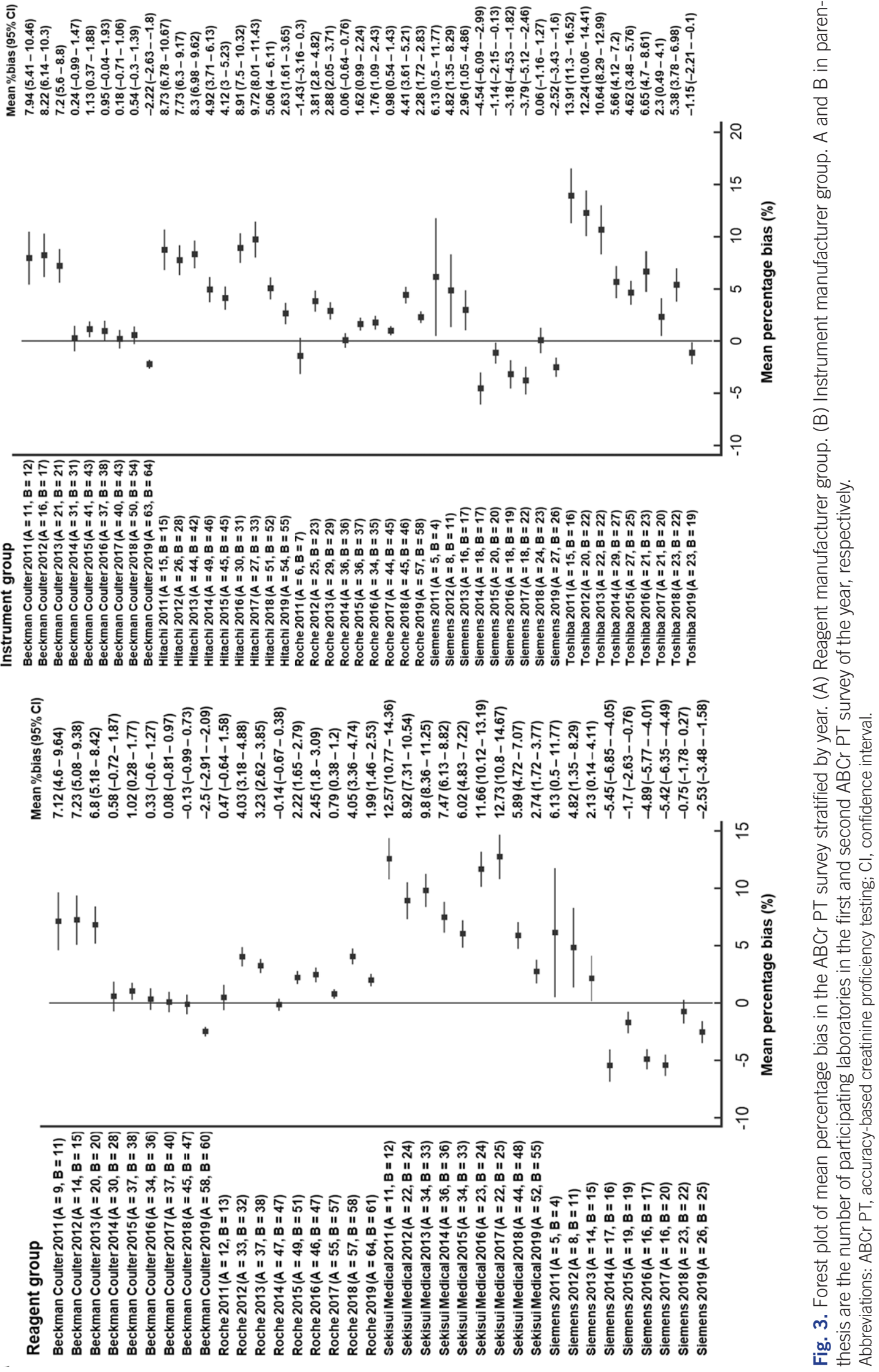
in 2016-2017 and resulted in more than a half of the participants using these reagents reporting "unacceptable" results for low-concentration samples. The main reason for the increased bias in this period was the relatively large number of low-concentration $(\mathrm{Cr}<1.0 \mathrm{mg} / \mathrm{dL})$ samples. Some laboratories discussed this issue with the manufacturer to decrease the bias and used compensated kinetic Jaffe assays to minimize the constant positive bias. As a result, the bias has decreased as of 2018 (i.e., mean \%bias: $11.7 \%$ in 2016 ; $12.7 \%$ in 2017 ; $5.9 \%$ in 2018 ; and $2.7 \%$ in 2019). This indicates that assay standardization can be greatly improved when clinical laboratories, manufacturers, and external quality assessment (EQA) agencies combine efforts.

The optimum, desirable, and minimum acceptable bias considering biological variation for $\mathrm{Cr}$ assays are $1.7 \%, 3.4 \%$, and $5.1 \%$, respectively, by the NKDEP and $1.9 \%, 3.7 \%$, and $5.6 \%$, respectively, by the latest European Federation of Clinical Chemistry and Laboratory Medicine data [16, 17]. ABCr PT survey participants using an enzymatic method had a mean percentage bias of $-3.9 \%-3.2 \%$ from 2011 to 2019 , satisfying the desirable bias criterion regardless of year and $\mathrm{Cr}$ concentration. Enzymatic methods avoid interference by Jaffe-like chromogens, such as glucose, protein, and acetone, allowing more accurate Cr concentration measurements than the Jaffe method [18, 19].

Participants who used non-enzymatic methods exceeded the minimum acceptable bias criterion in 2011 , with a $9.8 \%$ mean percentage bias, but generally met the desirable bias criterion after 2014. However, unlike the enzymatic method, bias significantly differed depending on the $\mathrm{Cr}$ concentration (i.e., $<1.0$ $\mathrm{mg} / \mathrm{dL}$ vs. $\geq 1.0 \mathrm{mg} / \mathrm{dL}$ ) for the non-enzymatic method. As of 2014, samples with a $\mathrm{Cr}$ concentration $\geq 1.0 \mathrm{mg} / \mathrm{dL}$ showed a $-1.3 \%-2.0 \%$ bias and usually met the optimum bias criterion, whereas samples with a $\mathrm{Cr}$ concentration $<1.0 \mathrm{mg} / \mathrm{dL}$ showed $3.6 \%-7.2 \%$ mean percentage bias, resulting in many cases not meeting the minimum acceptable bias criterion. Although the $\mathrm{Cr}$ bias for ABCr PT survey participants gradually decreases over time, the non-enzymatic method still overestimates $\mathrm{Cr}$ concentration and consequently underestimates eGFR, especially in low-concentration samples. Relatively larger bias in the Jaffe method than in the enzymatic method at low $\mathrm{Cr}$ concentrations has been reported [10, 13, 20].

Conversely, a negative bias tendency was observed in highconcentration samples. There was $-2 \%$ to $-3 \%$ negative bias in samples with a concentration $>2.0 \mathrm{mg} / \mathrm{dL}$. In the Italian study, the bias distribution in $227.2 \mu \mathrm{mol} / \mathrm{L}(2.57 \mathrm{mg} / \mathrm{dL})$ samples was $-8.4 \%$ to $-1.0 \%$ in 2011 [12]. Similarly, in the 2011 College of
American Pathologists survey, the bias for $2.77 \mathrm{mg} / \mathrm{dL}$ samples was $-4.9 \%-0.9 \%$, and that of $4.09 \mathrm{mg} / \mathrm{dL}$ samples was $-4.7 \%$ $0.4 \%$, revealing an overall negative bias [10]. The different bias tendencies between low- and high-concentration samples are assumed to be a calibration effect. Currently, the surest way to decrease bias in low-concentration samples and inter-laboratory variation is to use enzymatic $\mathrm{Cr}$ assays. However, this method has practical limitations, including high cost.

This study had some limitations. First, the $\mathrm{Cr}$ assay status in small laboratories was not represented. Second, although outliers were removed, the definition of an outlier lacks scientific rationale; the mean percentage bias in $\mathrm{Cr}$ concentration measurement for the participating laboratories in the KEQAS ABCr PT survey may vary depending on how the outlier is defined. Third, the bias for the detailed Jaffe method was not analyzed.

In conclusion, the long-term KEQAS ABCr PT survey demonstrated the $\mathrm{Cr}$ assay standardization status across participants and provided information for improving assay quality. Overall, the bias in $\mathrm{Cr}$ assay tends to decrease with participation in the KEQAS ABCr PT survey; however, continuous efforts are needed to decrease the bias for low-concentration samples. Clinical laboratories should consistently monitor $\mathrm{Cr}$ assay accuracy by using standardized calibrators and reagents that maintain metrological traceability, and by participating in an ABCr PT program. Coordinated efforts of clinical laboratories, manufacturers, and EQA agencies may further standardize $\mathrm{Cr}$ assay.

\section{ACKNOWLEDGEMENTS}

The KEQAS provided the ABCr PT survey data. We acknowledge all participating laboratories for their significant contribution to improving the quality of $\mathrm{Cr}$ assays in Korea.

\section{AUTHOR CONTRIBUTIONS}

TDJ designed the study, analyzed the data, and wrote the draft; WKM conceived the study, analyzed the data, and finalized the draft; EJC and KL discussed the data; WL, YMY, and SC discussed the data and reviewed the manuscript. All authors read and approved the final manuscript.

\section{CONFLICTS OF INTEREST}

None declared. 


\section{RESEARCH FUNDING}

None declared.

\section{ORCID}

Tae-Dong Jeong

Eun-Jung Cho

Kyunghoon Lee

Woochang Lee

Yeo-Min Yun

Sail Chun

Junghan Song

Won-Ki Min https://orcid.org/0000-0002-3873-3117 https://orcid.org/0000-0003-1907-9639 https://orcid.org/0000-0002-3154-0347 https://orcid.org/0000-0003-3956-6397 https://orcid.org/0000-0002-5485-8331 https://orcid.org/0000-0002-5792-973X https://orcid.org/0000-0003-0576-9938 https://orcid.org/0000-0002-5158-2130

\section{REFERENCES}

1. Stevens LA, Coresh J, Greene T, Levey AS. Assessing kidney functionmeasured and estimated glomerular filtration rate. N Engl J Med 2006; 354:2473-83.

2. Kidney disease: Improving Global Outcomes (KDIGO) CKD Work Group. KDIGO 2012 clinical practice guideline for the evaluation and management of chronic kidney disease. Kidney Int Suppl 2013;3:1-150.

3. Jeong TD, Cho EJ, Lee W, Chun S, Hong KS, Min WK. Accuracy assessment of five equations used for estimating the glomerular filtration rate in Korean adults. Ann Lab Med 2017;37:371-80.

4. Park $S$ and Jeong TD. Estimated glomerular filtration rates show minor but significant differences between the single and subgroup creatininebased chronic kidney disease epidemiology collaboration equations. Ann Lab Med 2019;39:205-8.

5. Kidney disease: Improving global outcomes (KDIGO) Acute Kidney Injury Work Group. KDIGO clinical practice guideline for acute kidney injury. Kidney Int Suppl 2012;2:1-138.

6. Levey AS, Coresh J, Balk E, Kausz AT, Levin A, Steffes MW, et al. National Kidney Foundation practice guidelines for chronic kidney disease: evaluation, classification, and stratification. Ann Intern Med 2003;139: 137-47.

7. Miller WG. The role of proficiency testing in achieving standardization and harmonization between laboratories. Clin Biochem 2009;42:232-5.

8. Miller WG, Jones GR, Horowitz GL, Weykamp C. Proficiency testing/ex- ternal quality assessment: current challenges and future directions. Clin Chem 2011;57:1670-80.

9. Jeong TD, Lee HA, Lee K, Yun YM. Accuracy-based proficiency testing of creatinine measurement: 7 years' experience in Korea. J Lab Med Qual Assur 2019;41:13-23.

10. Killeen AA, Ashwood ER, Ventura CB, Styer P. Recent trends in performance and current state of creatinine assays. Arch Pathol Lab Med 2013;137:496-502.

11. CLSI. Preparation and validation of commutable frozen human serum pools as secondary reference materials for cholesterol measurement procedures; approved guideline. CLSI C37-A. Wayne, PA: Clinical and Laboratory Standards Institute. 1999.

12. Carobene A, Ceriotti F, Infusino I, Frusciante E, Panteghini M. Evaluation of the impact of standardization process on the quality of serum creatinine determination in Italian laboratories. Clin Chim Acta 2014:427: 100-6.

13. Helmersson-Karlqvist J, Ridefelt P, Boija EE, Nordin G. Lower creatinine concentration values and lower inter-laboratory variation among Swedish hospital laboratories in 2014 compared to 1996: results from the Equalis external quality assessment program. Clin Chem Lab Med 2019. 57:838-44.

14. Levey AS, Coresh J, Greene T, Marsh J, Stevens LA, Kusek JW, et al. Expressing the Modification of Diet in Renal Disease Study equation for estimating glomerular filtration rate with standardized serum creatinine values. Clin Chem 2007;53:766-72.

15. Stevens LA, Manzi J, Levey AS, Chen J, Deysher AE, Greene T, et al. Impact of creatinine calibration on performance of GFR estimating equations in a pooled individual patient database. Am J Kidney Dis 2007;50: 21-35.

16. Myers GL, Miller WG, Coresh J, Fleming J, Greenberg N, Greene T, et al. Recommendations for improving serum creatinine measurement: a report from the Laboratory Working Group of the National Kidney Disease Education Program. Clin Chem 2006;52:5-18.

17. Aarsand A, Fernandez-Calle P, Webster C, Coskun A, Gonzales-Lao E, Diaz-Garzon J, et al. The EFLM Biological Variation Database. https://biologicalvariation.eu/ (Updated on 27 Apr 2020).

18. Cobbaert CM, Baadenhuijsen H, Weykamp CW. Prime time for enzymatic creatinine methods in pediatrics. Clin Chem 2009;55:549-58.

19. Piéroni L, Delanaye P, Boutten A, Bargnoux AS, Rozet E, Delatour V, et al. A multicentric evaluation of IDMS-traceable creatinine enzymatic assays. Clin Chim Acta 2011;412:2070-5.

20. Drion I, Cobbaert C, Groenier KH, Weykamp C, Bilo HJ, Wetzels JF, et al. Clinical evaluation of analytical variations in serum creatinine measurements: why laboratories should abandon Jaffe techniques. BMC Nephrol 2012;13:133. 